\title{
Theoretical Analysis and Simulation of Buffer Cylinder for Anti-Water Hammering
}

\author{
Guolai Yang ${ }^{1,2, a}$, Mingxue Li1, b , Guixiang Bai ${ }^{1, c}$, Meilin Xu1, d
}

${ }^{1}$ School of Energy and Power Engineering, Lanzhou University of Technology, Lanzhou, Gansu 730050, China;

2Lanzhou LS research institute, Lanzhou University of Technology, Lanzhou, Gansu 730050, China.

ayanggl@lut.cn, blimingxue1234@yeah.net, c554348543@qq.com, dxmlmnb@qq.com

Keywords: Water Hammer, Buffer Cylinder, Theoretical Analysis, Simulation.

\begin{abstract}
Aiming at the serious water hammer problem produced by check valve when the pump stops unexpectedly, an optimal method of using buffer cylinder to quick close check valve to reduce water hammer pressure is proposed. Through studying found that water hammer pressure is proportional to flow rate. And the buffer cylinder reduced the flow rate of check valve during the slow closing process, thus limiting the pressure of pipeline within an allowable range. In this paper, the theoretical analysis and AMESim simulation of the buffer process are designed to provide reference for further structural optimization.
\end{abstract}

\section{Introduction}

Check valve is mainly used for pipe system to prevent the back-flow of medium and protect pipes and machinery from water hammer damage. The instantaneous impact pressure of the water hammer $\Delta H$ may be several times higher than the normal working pressure during the working process [1], which has a great influence on the system performance stability and work reliability [2-3]. Now, damping buffer cylinder device is adopted to make valve fast off and slowly closed, to reduce the impact of water hammer [4-6].

\section{Check Valve's Working Principle}

The check valve is opened under the effect of static pressure and momentum of medium. When the valve is fully open, the valve flap is in a dynamic equilibrium state and it does not move with the change of media,s speed, and the flow resistance coefficient is minimum and constant. In the event of an accidental pump stop, the fluid accelerates in the opposite direction and the check valve quickly closes, causing severe water hammer pressure at the moment of shutdown, affecting the service life of the pipeline equipment.

\section{Structural Optimization Research}

\subsection{The Buffer Principle of Cylinder's Damping}

Cylinder cushioning device buffers and absorbs the kinetic energy of moving parts. The working principle is: when the buffer piston into buffer chamber, the oil between piston and cap squeezed out from hole or slit to produce a great resistance, the working part,s speed is gradually slow down by braking, so as to achieve the effect of deceleration and buffering[7-8].

\subsection{The Buffer Process of Cylinder's Damping}

Cylinder piston is pushed when check valve is opened under the action of the medium. Oil flows into right cavity through check valve, and valve flap is opened at a slow speed so as to avoid the impact of water hammer. Check valve opened when cylinder piston moved to the port $J$, the fluid quickly flows into right cavity, check valve,s opening speed is boosted, the medium,s flow resistance coefficient and pressure loss are reduced. The piston has blocked the port $N$ when the valve is fully opened, the oil at bottom of cylinder is sealed, to absorb the kinetic energy of piston, so as to prevent 
collisions. At the same time, preventing the valve opening angle is too large, leading to the immediate response can not shut down. The valve at the maximum position to maintain the balance, reducing the disturbance to flow regime and reducing the friction loss and fatigue damage at shaft [9].

Medium,s flow speed decreases when pump stop, the valve tends to close. By the tension and left chamber pressure the piston began to move, the oil at right cavity through the port $J$ quickly flow to the left chamber. Check valve quickly close most of the trip, resulting in a lot of local resistance, part of the water backwards, making the boost pressure decreases. Medium under gravity began to reverse flow, the piston blocked the port $J$, the oil can only flow from the orifice. Cavity oil pressure rise and resistance to the piston, the piston,s speed gradually reduced to zero, the check valve also slowly closed the remaining stroke. The increase of pressure is proportional to the change of flow rate, and the increase of flow rate decreases due to slow closing. The pipeline,s pressure increase is limited within a allowable range, which effectively reduces the water hammer of pump,s stop.

\subsection{Theoretical Analysis and Calculation}

Buffer cylinder structure diagram shown in Fig.1. This is a cushioning cylinder which uses automatic feedback of piston,s position to achieve cushioning. When piston moves to port $J$, the throttle starts to buffer. When the piston leaves the port $J$, the cylinder moves quickly.

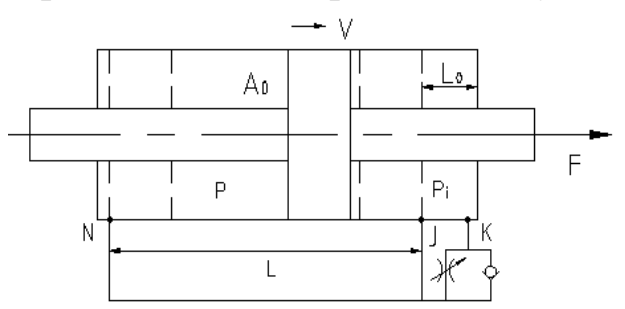

Fig.1 Structure diagram of buffer cylinder

Rightward movement of the piston speed to port $J$ is $v_{0}$,

$$
v_{0}=\frac{4 Q_{J}}{\pi A_{0}}
$$

In Eq. (1):

$Q_{J}$ - The flow of cylinder movement process from port $J$ out,

$A_{0}$ - The effective area of buffer piston.

Cylinder piston rapid movement phase time that is check valve fast closing time is $t_{1}$,

$$
t_{1}=\frac{L}{v_{0}}
$$

In Eq. (2):

$L \quad$ - The length of piston rapid movement phase.

Pistons arrive at the port $J$ began to buffer, set the total mass of piston and load as $m$, external force acting on the piston is $F$, then there is :

$$
F-F_{f}-\Delta P A_{0}=m \frac{d v}{d t}
$$

In Eq. (3):

$F$-External force acting on the piston,

$\Delta P$ - The pressure differential across buffer piston, $\Delta P=P_{i}-P$,

$F_{f}$ - Seal resistance (It,s related to the piston speed, sealing conditions, etc. and often overlooked when design and calculation),

$m$ - The quality of piston and moving parts,

$a$ - Piston, s acceleration.

Eq. (1) simplified as:

$$
F-\Delta P A_{0}=m \frac{d v}{d t}
$$

According to the continuity equation: 
In Eq. (5):

$$
Q=v \cdot A_{0}
$$

$V$-Piston, s speed,

$Q$ - The flow through throttle valve during damping.

The flow through throttle orifice is:

$$
Q=C_{q} A_{T} \sqrt{\frac{2}{\rho} \Delta P}
$$

In Eq. (6):

$C_{q}$ - Hole flow coefficient,

$A_{T}$ - Throttle flow area, $A_{T}=\frac{\pi}{4} d^{2}$,

$\rho$ - Fluid density.

From Eq. (4), Eq. (5) and Eq. (6) can be drawn:

$$
\begin{gathered}
a=\frac{d v}{d t}=\frac{1}{m}\left(F-\frac{A_{0}{ }^{3} V^{2} \rho}{2 C_{q}^{2} A_{T}{ }^{2}}\right) \\
\Delta P=\left(\frac{A_{0}}{C_{q} A_{T}}\right)^{2} \rho \cdot \frac{v^{2}}{2}=\frac{F}{A_{0}}-\frac{m}{A_{0}} \frac{v d v}{d x}
\end{gathered}
$$

In Eq. (8):

$x$ - Buffer piston,s displacement.

Piston displacement can be obtained by integration:

$$
x=\int_{0}^{x} \frac{2 C_{q}^{2} A_{T}^{2} m v}{2 F C_{q}^{2} A_{T}^{2}-\rho A_{0}^{3} v^{2}} d v=-\frac{A_{T}^{2} C_{q}^{2} m \ln \left(A_{0}^{3} \rho v^{2}-2 A_{T}^{2} C_{q}^{2} F\right)}{A_{0}^{3} \rho}
$$

Boundary conditions, $x=0, v=v_{0}$, the throttle began to buffer. To simplify,make $\frac{2 A_{T}{ }^{2} C_{q}{ }^{2}}{A_{0}^{3} \rho}=k$,

$$
v=\left(\frac{e^{-\frac{x}{k m}}+2 A_{T}^{2} C_{q}^{2} F}{A_{0}^{3} \rho}\right)^{1 / 2}
$$

Substituting boundary conditions, Eq. (9) and Eq. (10) into Eq. (7),

$$
a=\frac{d v}{d t}=-\frac{F}{m}\left(\frac{v_{0}{ }^{2}}{v_{\infty}{ }^{2}}-1\right) e^{-\frac{2 F x}{m v^{2}}}
$$

When $x=0$, the deceleration was $a=a_{\max }$,

Deceleration general expressed as:

$$
a=a_{\max } \cdot e^{-\frac{2 F x}{m v_{\infty}^{2}}}
$$

Substituting Eq. (10) into Eq. (8), can obtain:

$$
\Delta P=\frac{e^{-\frac{x}{k m}}+2 A_{T}^{2} C_{q}^{2} F}{2 A_{0} C_{q}^{2} A_{T}^{2}}
$$

The buffer chamber,s pressure is maximum, when $x=0$.The buffering process ends, when deceleration $a=(1 \sim 5) \% a_{\max }[8]$.

From Eq. (7) and Eq. (8), can obtain:

$$
\begin{gathered}
d t=\left(\frac{e^{-\frac{x}{k m}}+2 A_{T}^{2} C_{q}^{2} F}{A_{0}^{3} \rho}\right)^{-1 / 2} d x \\
t_{0}=-k m \cdot\left(-F A_{T}^{2} C_{q}^{2}\right)^{-1 / 2} \cdot \arctan \left(\frac{\left(2 F A_{T}^{2} C_{q}^{2}+e^{-\frac{x}{k m}}\right) A_{0}^{3} \rho}{-F A_{T}^{2} C_{q}^{2}}\right)^{1 / 2}
\end{gathered}
$$


$t_{0}$ - It,s the time when cylinder acts as a buffer and also the time it takes for check valve to close slowly.

$$
L_{0}=x_{\max }=k_{l} \frac{m v_{\infty}^{2}}{F}
$$

$k_{l}$ - Length factor, make $k_{l}=1.5 \sim 2.3$ [8].

The water hammer increased pressure is different when water hammer occurred at check valve which have different types and sizes [10]. The theoretical analysis formula above is used to calculate the size of damping orifice, the buffer speed and two stages of valve closing time, making the close process of check valve is coordinated with the transition state of water flow, so as to control water hammer pressure effectively.

\section{Simulation}

The simulation model of hydraulic buffer cylinder is established by AMESim simulation environment [11-12], and the simulation is carried out. The first stage of buffer cylinder is the ordinary cylinder's rapid movement, so only consider the simulation of buffer process, that is, the simulation of buffer process after piston plug port $J$.

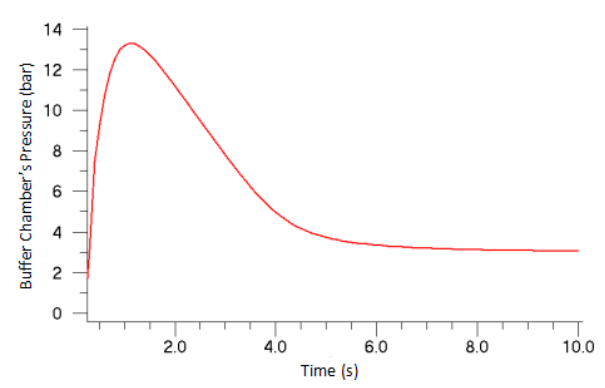

Fig.2 Pressure curve of buffer chamber

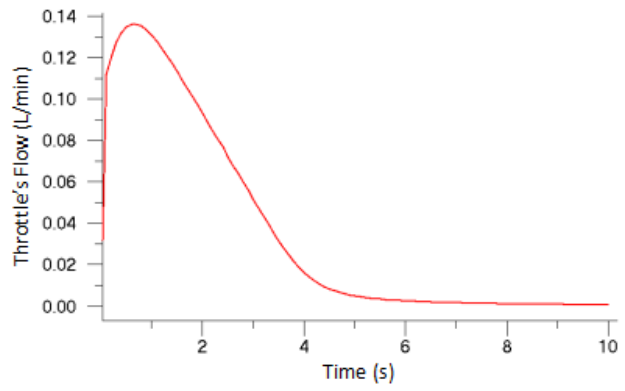

Fig. 3 Flow curve of throttle

Fig. 2 shows the pressure curve of buffer chamber. When the maximum back-flow medium arrives at the check valve, the oil pressure in the closed cylinder of the cylinder gradually increases, causing resistance to moving piston. The piston speed gradually decreases to zero, and the buffer makes check valve closing time increased.

Fig. 3 is the flow curve of throttle. It can be seen from Fig. 3 that: the oil flows out of throttle when piston blocks port $J$, and the buffering phase ends when flow rate decreases to zero.

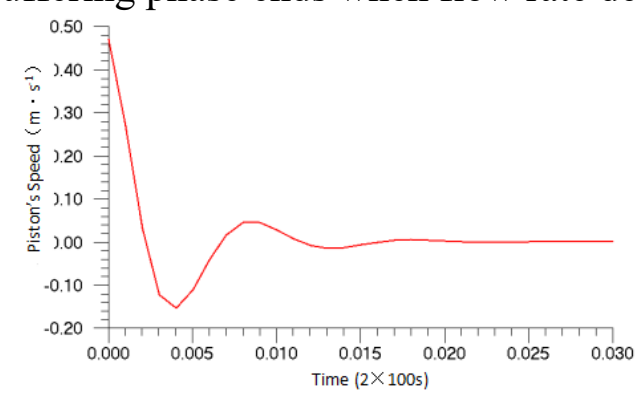

Fig.4 Speed curve of sports piston

Fig. 4 is the speed curve of sports piston. It shows: damping buffer cylinder piston speed slowly reduced to zero, extending the check valve slow closing process time, thus limiting the value of water hammer pressure increase within an allowable range. Check valve closing pressure depends on its dynamic shut-off feature [13-14], improves the structure can reduce the water hammer strength.Anti-water hammering buffer cylinder's performance is verified by theoretical analysis and simulation.

\section{Conclusion}

(1) Buffer cylinder overcomes the shortcomings of ordinary check valve, making check valve to achieve the function of fast closing and slow closing. And the close process of check valve is 
coordinated with the transition state of water flow, so as to control water hammer pressure effectively.

(2) Theoretical analysis can help to select a certain range of water hammer pressure increase corresponding to the size of the buffer cylinder,s damping orifice.

(3) Through theoretical analysis and simulation of buffer cylinder, the feasibility of buffer cylinder is verified.

(4) The effect of buffer cylinder effectively prevents the pressure increase of water hammer in check valve, protects pumps and pipeline systems, and affects the movement of medium in check valve, thus providing a reference for engineering design and optimization of piping system.

\section{References}

[1] Wanglong Lu. Hydraulic System Service and Repair Manual [M] .2008.

[2] Wilkinson D H, Dartnell L M. Water Hammer Phenomena in Thermal Power Station Feed Water System. Proc. Inst. of Mech. Eng., Vol. 194, Jan. 1980.

[3] Bin Liu. Hazard and Protection of Water Hammer in Pump [J]. Shanxi Building Science, 2009, 35 (24): 182.

[4] Qingyun Yao. The Water Hammer Control Protection with Two-stage Closing Valve at Pumping Station [J]. Journal of Ningxia Agricultural College, 1997, 18 (1).

[5] Liming Yang, Xiuyun Wu, Nianshen Wang. Study on the Protection of Water Hammer with Slow Closing Check Valve. Chinese Journal of Safety Science [J], 2004, 14 (11).

[6] Yingfeng Chen. Development of Spin-on Buffer Check Valve. Petrochemical Equipment Technology [J], 1998, 19 (1): 39.

[7] Bo Liu. Cushion Structure and Cushioning Process of Hydraulic Cylinder [D] .2004.

[8] Cunxing He, Jianya Lin. Hydraulic Components [M]. 198812 (18): 271.

[9] Peng Yue, Yanjun Wang, Jinliang Liu, et al. Analysis of Anti-Fatigue Performance of Nuclear Valves [J]. Valve, 2009, 1: 30.

[10]Provoost G A, A Critical Analysis to Determine Dynamic Characteristic of Non2return Valves. 4th Int. Conf. on Pressure Surges, 1983.

[11]Zhijun Luo, Jian Xie, Gui Tian.Study on Variable-load Synchronization Control of Multi-stage Hydraulic Cylinder. Fluid Transmission and Control [J], 2009, 4 (35).

[12] Qiuhong Chang, Shimin Yang, Xuan Li. Optimization Method of Bucket Arm Cylinder for Mine Vertical Claw Loader. Applied Science and Technology [J], 2011, 38 (8).

[13] Li Yongde. Study on Dynamic Characteristics of Check Valve. Fluid Machinery, 1994, 22 (8): 7.

[14] F M, AD H, NH Hang. Dynamic Characterization of a New Accelerated Heart Valve Tester [J]. ASAIO JOURNAL,1997,43(5):M372-M37 